\title{
A new high sensitivity radar sensor for space debris detection and accurate orbit determination
}

\author{
Alessandro Morselli, Pierluigi Di Lizia \\ Politecnico di Milano - Department of Aerospace Science \\ and Technology \\ Via La Masa 34, 20156 Milano, Italy
}

Germano Bianchi, Claudio Bortolotti, Stelio

Montebugnoli, Giovanni Naldi, Federico Perini, Giuseppe Pupillo, Mauro Roma, Marco Schiaffino, Andrea Mattana, Emma Salerno.

Istituto Nazionale di Astrofisica - Istituto di Radioastronomia (INAF-IRA)

via Fiorentina 3513, 40059 Medicina (BO), Italy

\author{
Alessio Magro, Kristian Zarb Adami \\ University of Malta \\ Msida MSD 2080, Malta
}

\author{
Roberto Armellin \\ University of Southampton - Astronautics and \\ Computational Engineering Academic Unit \\ Highfield Campus, Southampton, SO17 1BJ, UK \\ Andrea Lazzareschi Sergiusti \\ Vitrociset S.p.A. \\ Via Tiburtina 1020, 00156 Rome, Italy \\ Walter Villadei, Ferdinando Dolce, Marco Reali, \\ Jacopo Paoli \\ Italian Air Force (ITAF) \\ Viale dell'Università 4, 00185 Rome, Italy
}

\begin{abstract}
As the amount of space debris orbiting the earth is continuously increasing, it is becoming essential to monitor and predict the debris' trajectories in order to avoid collision that could threaten space missions, i.e. operative satellites or manned spacecraft. An innovative bistatic radar system, that couples multi-beaming and ranging techniques, is proposed in this work as an instrument for the observation of Earth-orbiting objects. The main characteristics of the sensor are described. In addition, tailored algorithms have been implemented to perform orbit determination using the peculiar outputs provided by the sensor. The results of numerical simulations are illustrated to assess its performances for space debris detection and orbit determination.
\end{abstract}

Keywords-Multibeam; beamforming; bistatic radar; orbit determination; space debris; pulse compression.

\section{INTRODUCTION}

Ground-based radars provide a powerful tool for the characterization of the orbital debris environment. Radars can in fact irradiate at any time a satellite or space debris in Earth orbit with a microwave beam. The scattered wave can be detected by a receiver, which may be the same transmitting antenna (monostatic radar) or a different one located at a distance of up to several hundreds of kilometers away (bistatic radar).

A novel bistatic radar sensor is proposed in this work, which makes use of a multibeam receiver. Unlike standard radar sensor configurations, multibeaming enables the characterization of the transit direction of the scattering object inside the field of view (FoV) of the receiver. The information about the sequence of beams that are illuminated indeed allows to discern the trajectory of the object in terms of right ascension and declination (or alternatively in terms of azimuth and elevation).

\section{BISTATIC ARCHITECTURE}

The proposed sensor uses the "Flight Termination System" (FTS) of Italian Joint Test Range of Salto di Quirra (PISQ) in Sardinia, as the transmitter part (fig. 1), and the Northern Cross radiotelecope of the radioastronomy station of Medicina (BO) as the receiver part (fig. 2).

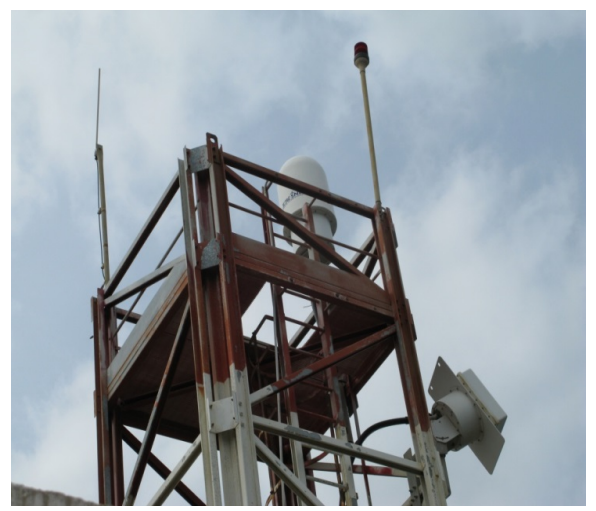

Fig. 1. The "Flight Termination System" (FTS) transmitter.

The FTS is a powerful transmitter, owned by Italian Air Force, presently used for safety purposes during the system trials at PISQ. The FTS consists in a power amplifier able to supply an average and leveled power of $4 \mathrm{~kW}$ in the bandwidth 400-455 MHz coupled with an Omnidirectional Antenna and wide beam directional antenna. The transmitter is usually used in $\mathrm{CW}$ mode, anyway the equipment is able to receive in input 
a modulated signal. Clearly the use in $\mathrm{CW}$ mode does not enable the bi-static sensor to detect the range of the object, whereas using a dedicated, synchronized waveform and applying the right modulation signal enables the ranging capability.

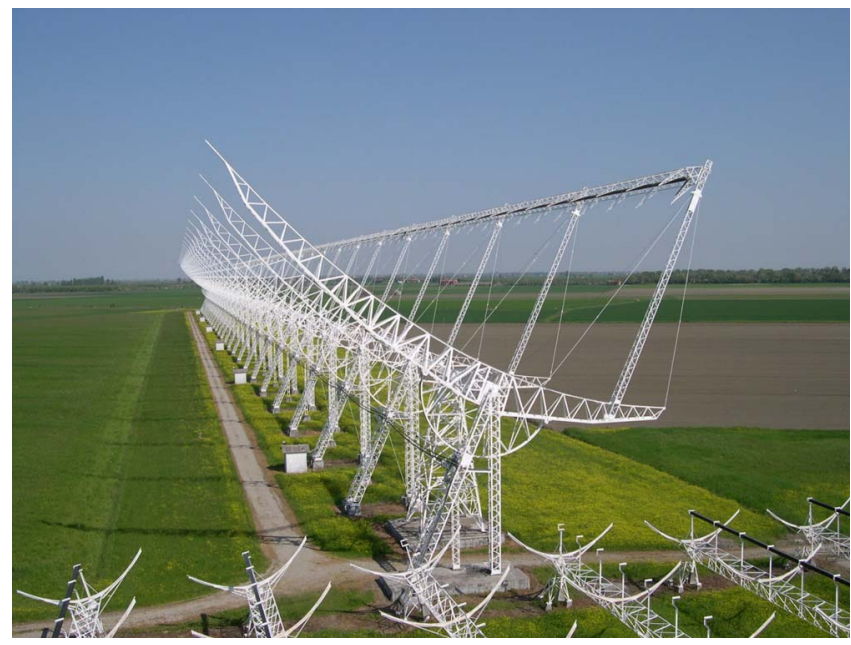

Fig. 2. A part of the Northern Cross radiotelescope.

The Northern Cross represents the largest UHF-capable antenna in Northern hemisphere. It is composed of two perpendicular arms: the E/W (East-West) arm is $564 \mathrm{~m}$ long and consists of a single cylindrical antenna with a width of 35 $\mathrm{m}$, whereas the N/S (North-South) arm is made of 64 parallel antennas with a length of $23.5 \mathrm{~m}$ and a width of $8 \mathrm{~m}$ each. The collecting area reaches $27,000 \mathrm{~m} 2$ and, due to the large numbers of receivers which could be installed on the focal lines, the FoV can be populated with many independent beams. When an object transits inside the antenna FoV, beams are illuminated by the reflected radio wave. By looking at the beam illumination sequence, it is thus possible to estimate the ground track of the transiting object, with a higher level of detail with respect to a single-beam system (fig. 3). The acquired data is processed by means of a data acquisition system, which digitally assembles measured radar echoes using an FFT in spatial domain in order to calculate the signal present in each beam [1]. Doppler shift, the illumination time, and measured power intensity associated to each beam are thus available.

The same bistatic architecture could be used to perform ranging, provided that the transmission signal is modulated with pulse compression waveforms, transmitter and receiver are synchronized and the data processing chain contains a ranging estimation block. The bistatic radar shall use a ranging waveform based on pulse compression technique. The bandwidth needed to obtain the requested ranging performances is largely inside the transmitter and receiver characteristics. A frequency modulated signal shall be generated in the expander unit, up converted and sent to the transmitter. A high duty cycle transmission pattern (very long pulses at high repetition rate) shall supply an average power sufficient to guarantee detection and range extraction at the requested range. The transmitted waveform and its matched filter in the receiving branch shall be designed to obtain a good sidelobes level taking into consideration the Doppler shift due to the target motion. Pulse compression shall be performed digitally in the receiver part and the resulting ambiguous video shall be sent to range extraction algorithms. Range ambiguity shall be solved taking into consideration the geometry and a rough target range estimate.

The system can quickly switch from the multi-beaming observation mode (performed in continuous wave) to ranging mode: during the transit of an object both types of measurement can be thus acquired. The signals scattered from the object are then processed to estimate the orbital parameters. Tailored Orbit Determination (OD) algorithms were developed so that, using both the ranging and the beam illumination sequence, the orbital parameters of the transiting object can be estimated [2]. By combining this information with the knowledge of the beam distribution and antenna pointing, it is possible to refine the orbital parameters of known objects or to perform a preliminary OD.

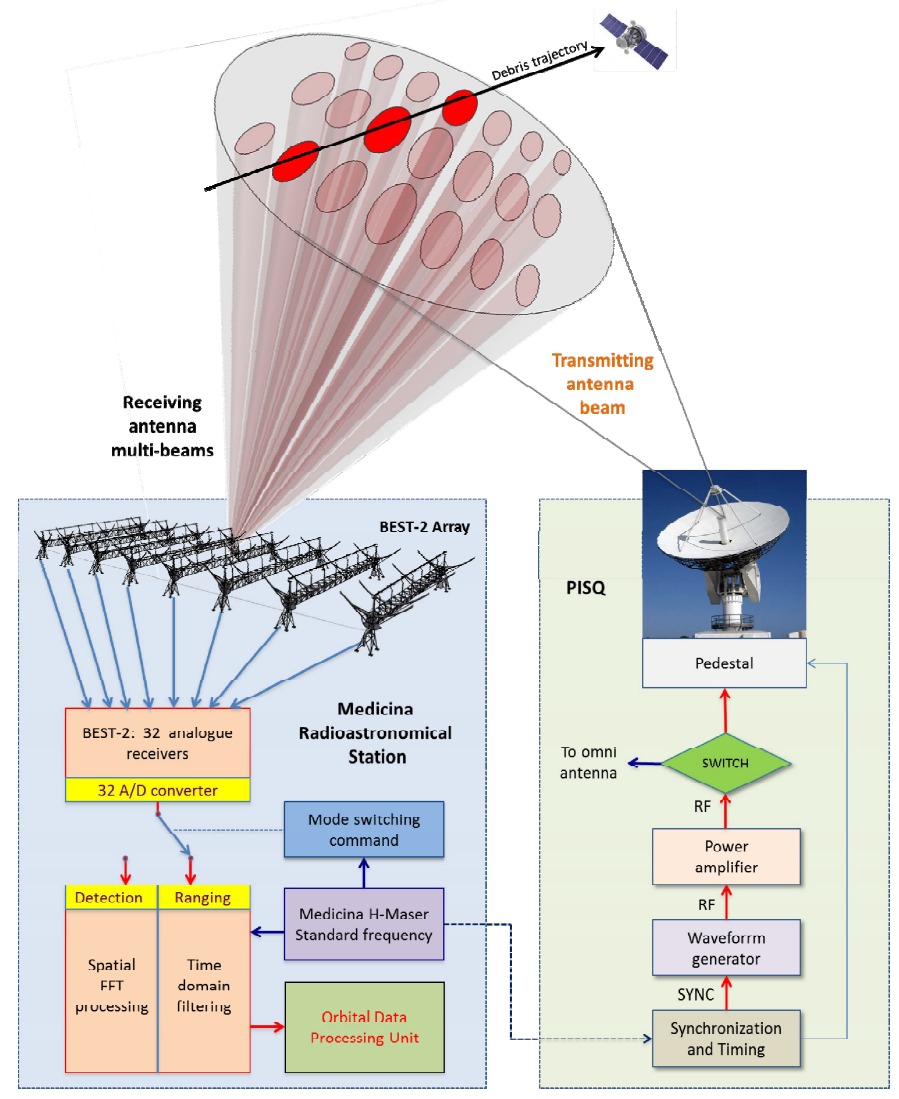

Fig. 3. Schematic block and example of space debris trajectory detection using a multibeams bistatic radar system.

\section{SYSTEM PERFORMANCE}

Due to the high sensitivity, large FoV and high number of sensors, the Northern Cross is able to detect lots of debris with very small dimension and to calculate their trajectory with extreme precision. At present, no other instruments exist with such a sensitivity and accuracy. 
The bi-static radar system proposed in this work will be a preliminary configuration to fulfill the basic requirements for the participation to the European SST (Space Surveillance and Tracking) Support Framework [3], and in particular to supply the detection of objects in LEO.

An existing transmitter upgraded with a state of the art digital waveform generator and a wide parabolic antenna $(7 \mathrm{~m}$ of diameter) capable to track the receiver multibeam pattern is foreseen. As a receiver, only a small part of the Northern Cross receiving antenna, named BEST-2 [4] (Basic Element for Ska ${ }^{1}$ Training) will be used (fig. 4). BEST-2 is an array composed of 8 cylindrical parabolic concentrators belonging to the N-S arm operating in a band of $14 \mathrm{MHz}$ centered at $408 \mathrm{MHz}$. The total collective area is about $1,400 \mathrm{~m} 2$. Each cylinder contains four receivers connected to 16 dipoles each (fig. 5).

The total array contains 32 receivers that enable the realization of a multibeam receiver antenna. More specifically, 24 independent beams (pixels) are generated within the antenna FoV using 32 receivers. A MATLAB ${ }^{\circledR}$ simulation of the BEST-2 pixelated FoV is shown in fig. 6. Considering the peak power of $5 \mathrm{~kW}$ deliverable by the transmitter, a $7 \mathrm{~m}$ Tx-dish, the BEST-2 antenna as receiver and a slant range of 2,000 km, the minimum detectable Radar Cross Section (RCS) is less than $1 \mathrm{~m} 2$. Detailed characteristics are reported in tab. 1 .

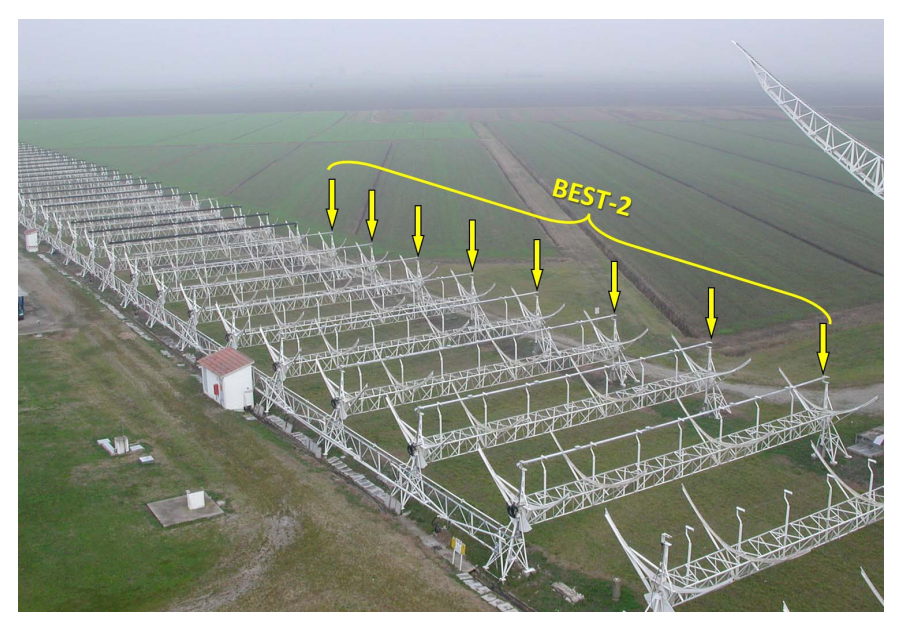

Fig. 4. BEST-2 antennas.

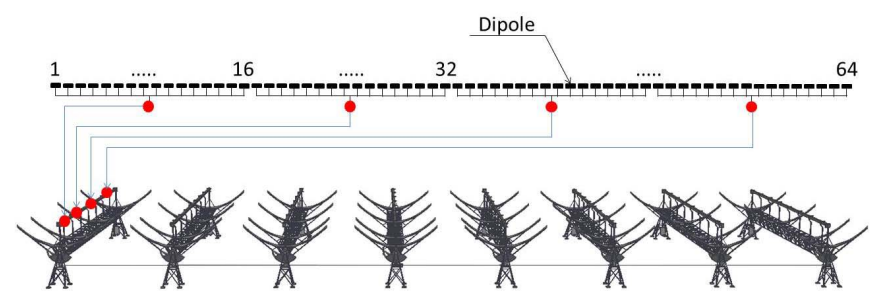

Fig. 5. Single BEST-2 antenna architecture (red points correspond to the receivers).

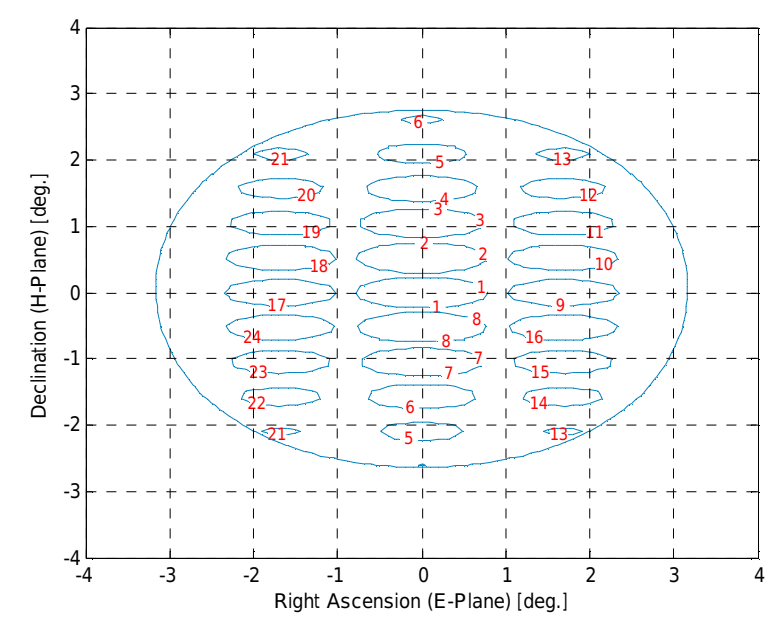

Fig. 6. BEST-2: FOV and Synthetized Beam allocation.

TABLE I. BEST-2 SYSTEM CARACTERISTICS

\begin{tabular}{|c|c|}
\hline Frequency (center band) & $408 \mathrm{MHz}$ \\
\hline Instantaneous bandwidth & $14 \mathrm{MHz}$ \\
\hline Mechanical elevation pointing & $>45 \mathrm{deg}$ \\
\hline Electrical azimuth pointing & $\begin{array}{c}-3.3 \mathrm{deg} \div 3.3 \mathrm{deg} \\
176.7 \mathrm{deg} \div 183.3 \mathrm{deg}\end{array}$ \\
\hline Instantaneous FoV & $\begin{array}{c}37.6 \text { degrees } 2 \\
(\text { Dec } 5.7 \mathrm{deg}, \text { AR } 6.6 \mathrm{deg})\end{array}$ \\
\hline Synthetized beam (pixel) & $\begin{array}{c}0.9 \text { degrees2 } \\
\text { (Dec } 31.1 \text { arcmin, AR } 104 \text { arcmin) }\end{array}$ \\
\hline Number of independent beams & 24 \\
\hline Minimum detectable RCS & $\begin{array}{c}0.32 \mathrm{~m} 2 \\
\text { (considering a slant range of } 2,000 \mathrm{~km} \text { ) }\end{array}$ \\
\hline
\end{tabular}

The data received from the BEST- 2 processing unit are given as input to the orbital data processing unit, which is aimed at providing an estimate of the orbital parameters of the observed object to be included in the catalogue and later used for conjunction assessment. In addition, the unit computes the uncertainty associated to the orbital state, usually expressed in terms of covariance matrix, which is the input required by any method to perform impact probability computation.

The entire orbit determination process is based on two main phases:

- Phase 1:Observables estimation. The topocentric right ascension and declination profiles are estimated starting from the Signal to Noise Ratio (SNR) profiles available for each beam;

- Phase 2: Orbit determination. The object position and velocity are estimated from the observables by matching the generated orbital trajectory with the range measurements, and the right ascension and declination profiles computed from Phase 1, and the recorded Doppler shift.

A scheme of the workflow of the orbital data processing unit is given in fig. 7. The objective of the observables estimation process (Phase 1) is to extract all the information 
that can be later fed to the orbit determination (Phase 2). The entire orbital data processing unit takes as input the data coming from the back-end and the bistatic radar geometry, including:

- Observation epoch

- Range measurement

- Signal to Noise Ratio (SNR) for each synthetized beam

- Doppler shift

- Receiver and transmitter pointing angles

The observables estimation block elaborates the information of SNR to extract the time profile of right ascension and declination of the satellite. The range information is also used to improve the estimate of the two angles.

Following the observables estimation process, all observables that can be obtained with the proposed bistatic radar configuration are available:

- Observation epoch

- Range measurement

- Right ascension and declination and their derivatives

- Doppler shift

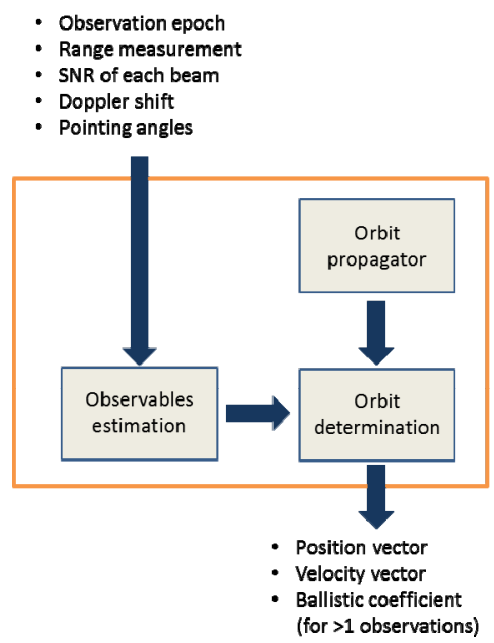

Fig. 7. Orbital data processing unit.

The above information is then transferred to the orbit determination block that estimates and matches object position and velocity with the observables. An orbit propagator is needed for this purpose. The current version of the algorithm uses the Accurate Integrator for Debris Analysis (AIDA) developed by Politecnico di Milano, which is based on the numerical integration of the dynamics of Earth-orbiting objects, including:

- the gravitational model EGM2008, up to the order specified by the user;

- the atmospheric drag with the atmosphere model NRLMSISE-00 to compute air density;

- third body perturbations;

- solar radiation pressure with a dual-cone model for Earth shadow.

\section{RESULTS ACHIEVED AND THEIR APPLICABILITY}

Numerical simulations involving LEO (Low Earth Orbit), HEO (Highly Elliptical Orbit) and GTO (Geostationary Transfer Orbit) objects are performed to analyze the performances of the system. Simulated data are generated and used to feed the OD algorithms. The resulting orbital parameters are then compared with those used to generate the simulated observations to assess the accuracy of the estimation. In this way the performances of the algorithms may be tested and the effectiveness of this innovative configuration for space debris measurements, that couples a bi-static radar, a multibeaming receiver, and radar ranging, can be assessed.

The simulations of the performances of the bistatic radar system described above are based on Two-Line Elements (TLEs) included in the public orbital data catalog provided by USSTRATCOM. $^{2}$ Since the number of detectable objects depends on the antenna pointing, we considered an intermediate case, where the BEST-2 receiving antenna elevation is $60 \mathrm{deg}$ with azimuth of $180 \mathrm{deg}$. The results show the capability to observe hundreds of objects in LEO (including also the set of HEO and GTO objects whose orbit crosses the LEO region) within a week (fig. 8). Note that the detection of the space objects depends on their transit in the common volume covered by the transmitter and receiver beams. The capability "to effectively detect them in a period of time" is therefore probabilistic.

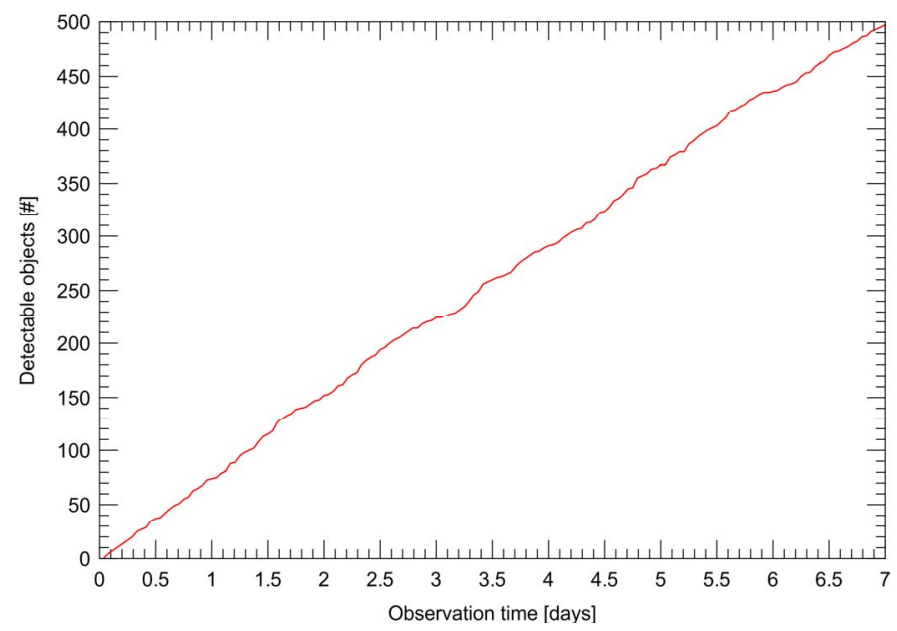

Fig. 8. Number of object detectable in LEO, HEO and GTO vs observation time.

A simulator of the proposed sensor was developed to support the analysis and estimate the OD performances. More specifically, given any object of interest, its reference trajectory is first computed using the TLEs and states derived by them as pseudo observations for an OD process. In this case a TLE is propagated backward and forward around the TLE epoch using SGP4/SDP4 to generate a set of Cartesian pseudo-observations. Then, these are used to solve an OD problem (typically a least-square problem), producing an estimate of the reference orbital state and trajectory, as well as the associated covariance matrix. The reference orbital state is

2 Available at www.space-track.org 
then propagated, and the simulator identifies its passages inside the instrument $\mathrm{FoV}$ and generates the inputs to the orbital data processing unit. The simulator is designed so that different kind of transmitter and multibeam geometry can be easily defined by the user. This enables an effective analysis of the sensitivity of the sensor to its configuration, which is instrumental to optimizing its performances.

An example of the performances achieved is reported hereafter. Simulated bistatic radar observations of two objects, with RCS of $6.5 \mathrm{~m} 2$ (object ID 19046) and $1.1 \mathrm{~m} 2$ (object ID 31140) respectively, were generated for the proposed sensor. The simulated tracklet of object 31140 , i.e. the trajectory covered by the satellite or debris inside the FoV of the antenna, is illustrated in fig. 9 (black line). The blue and red lines in the same figure are the first and refined estimates, respectively, of the tracklet, obtained within Phase 1 of the OD process. The red line is almost overlapping with the black one, which is the reference $(\alpha-\delta)$-trajectory for the OD process. The accuracy of the orbit determination is confirmed in fig. 10, which reports the profile of the error of the refined estimate with respect to the reference tracklet for the same object. The resulting maximum error on the estimation of the values of $\alpha$ and $\delta$ is $0.027 \mathrm{deg}$ and $0.019 \mathrm{deg}$, respectively.

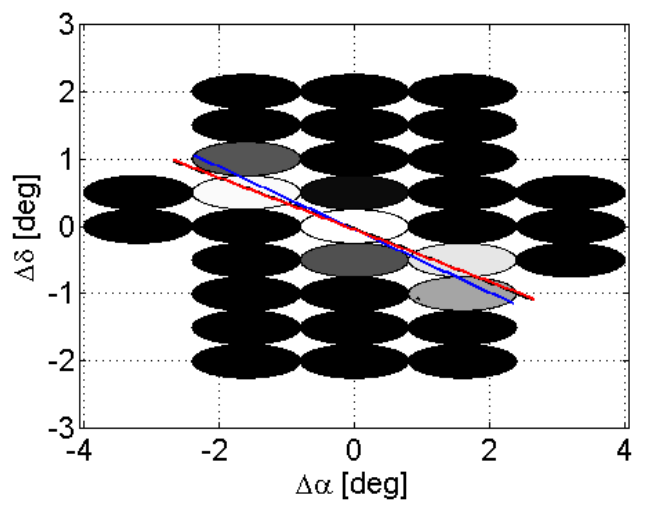

Fig. 9. Simulated receiver tracklet. The black line is the reference trajectory, the blue line the first estimate of the trajectory in the $(\alpha, \delta)$-plane, and the red line the refined $(\alpha, \delta)$-trajectory. The red line essentially coincides with the black one.

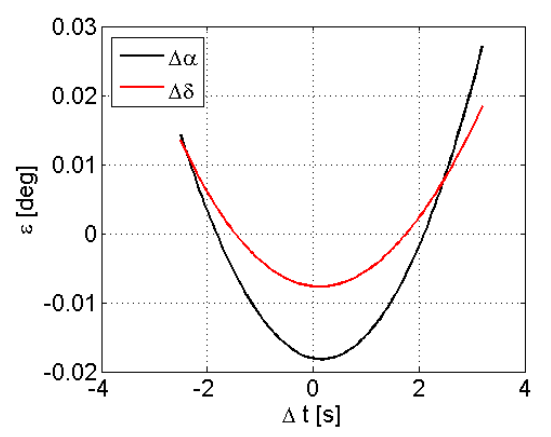

Fig. 10. Profiles of the error of the estimated right ascension $\alpha$ and declination $\delta$ with respect to the reference tracklet for object 31140 .

The reference and estimated object position and velocity at the end of Phase 2 of the OD process are reported in tab. 2 for both objects. These values were obtained by using the time of reception of the last echo as reference epoch for the orbit determination process. The reference object state corresponds to the reference trajectory provided by the simulator. The error on the position components is equal to a few tens of meters, whereas the error on velocity components reaches $1-10 \mathrm{~m} / \mathrm{s}$. The uncertainty on position is on the order of a few tens of meters and the ones on velocity are around $1 \mathrm{~m} / \mathrm{s}$.

TABLE II. ESTIMATED POSITION AND VELOCITY FOR TWO DIFFERENT OBJECTS, WITH RCS OF $6.5 \mathrm{M} 2$ AND $1.1 \mathrm{M} 2$ RESPECTILELY

\begin{tabular}{|c|c|c|c|c|c|c|}
\hline & $\begin{array}{c}\mathbf{R X} \\
{[\mathrm{km}]}\end{array}$ & $\begin{array}{c}\mathbf{R Y} \\
{[\mathrm{km}]}\end{array}$ & $\begin{array}{c}\mathbf{R Z} \\
{[\mathrm{km}]}\end{array}$ & $\begin{array}{c}\mathbf{V X} \\
{[\mathrm{km} / \mathrm{s}]}\end{array}$ & $\begin{array}{c}\mathbf{V Y} \\
{[\mathrm{km} / \mathrm{s}]}\end{array}$ & $\begin{array}{c}\mathbf{V Z} \\
{[\mathrm{km} / \mathrm{s}]}\end{array}$ \\
\hline \multicolumn{7}{|c|}{ Object 19046, reference epoch 01/07/2014 19:52:12:20 UTC } \\
\hline $\begin{array}{l}\text { Ref. } \\
\text { state }\end{array}$ & -3208.888 & -3746.830 & 4851.438 & 2.345987 & 4.901517 & 5.318343 \\
\hline $\begin{array}{c}\text { Est. } \\
\text { state }\end{array}$ & -3208.933 & -3746.789 & 4851.440 & 2.347366 & 4.900858 & 5.318389 \\
\hline \begin{tabular}{|c|} 
St. Dev. \\
$\sigma$ \\
\end{tabular} & \pm 0.014 & \pm 0.016 & \pm 0.021 & $\pm 1.578 \mathrm{e}-3$ & $\pm 1.041 \mathrm{e}-3$ & $\pm 0.537 \mathrm{e}-3$ \\
\hline \multicolumn{7}{|c|}{ Object 31140, reference epoch 01/07/2014 19:52:12:20 UTC } \\
\hline $\begin{array}{l}\text { Ref. } \\
\text { state }\end{array}$ & 3755.766 & 3243.782 & 4687.046 & -3.019328 & 6.667812 & -2.207117 \\
\hline $\begin{array}{l}\text { Est. } \\
\text { state }\end{array}$ & 3755.818 & 3243.754 & 4687.017 & -3.034418 & 6.664348 & -2.198952 \\
\hline \begin{tabular}{|c|} 
St. Dev. \\
$\sigma$ \\
\end{tabular} & \pm 0.034 & \pm 0.028 & \pm 0.026 & $\pm 3.059 \mathrm{e}-3$ & $\pm 1.236 \mathrm{e}-3$ & $\pm 1.674 \mathrm{e}-3$ \\
\hline
\end{tabular}

\section{CONCLUSION}

The work described in this paper shows a summary of the performance achievable by the bistatic radar proposed for the space debris monitoring. The system permits to detect orbiting objects of very small Radar Cross Section and to measure the range and angular information necessary to calculate and/or update space objects trajectories. This capability is important to monitor space debris and extract information to provide SST Data in LEO. The bistatic radar is a candidate to participate as a National asset at the European SST Support Framework.

The architecture is very flexible and modular and is suitable to improve the performance using added parts and upgrading both the Northern Cross antenna, whit relevant processing, and the Flight Termination System.

Tailored orbit determination algorithms have been developed to process sensor measurements and estimate the orbit of the observed space debris. Numerical simulations show that accurate results can be obtained and the orbital state of real objects could be retrieved with just a single pass, which paves the way to possibly use the sensor for real-time orbit determination.

\section{ACKNOWLEDGMENT}

The Flight Termination System is a facility of the Italian Air Force, located at Italian Joint Test Range of Salto di Quirra in Sardinia.

The Northern Cross Radio Telescope is a facility of the University of Bologna operated under agreement by the IRAINAF (Radio Astronomy Institute - National Institute of AstroPhysic). 


\section{REFERENCES}

[1] G. Foster, J. Hickish, A. Magro, D. Price and K. Zarb Adami, "Implementation of a direct-imaging and FX correlator for the BEST-2 array". Published by Oxford University Press on behalf of the Royal Astronomical Society.

[2] Morselli A., Armellin R., Di Lizia P., Bernelli Zazzera F., Salerno E., Bianchi G., Montebugnoli S., Magro A., Adami K.Z., "Orbit Determination of Space Debris Using a Bi-static Radar Configuration with a Multiple-Beam Receiver", In 65th International Astronautical Congress (IAC 2014), 29 September - 3 October, Toronto, Canada, IAC14-A6.9.4 (2014)
[3] Ddecision N. 541/2014/EU of the European Parliament and of the council, 16 April 2014.

[4] S. Montebugnoli, G. Bianchi, J. Monari, G. Naldi, F. Perini, M. Schiaffino, "BEST: Basic Element for SKA Training". Wide Field Science and Technology for the Square Kilometre Array, Proceedings of the SKADS Conference held at the Château de Limelette, Belgium, 3-6 November 2009, p. 331-336.

[5] Italian Air Force - National Institute of Astrophysics (ITAF - INAF) "Agreement for Space Situational Awareness Experimentation", May 2013. 